\title{
Experimental comparison of FBARs and SMRs responsitivities to mass loadings
}

\author{
L. García-Gancedo ，J. Pedrós , E. Iborra , M. Clement , J. Olivares , J. Capilla , \\ J.K. Luo , W.I. Milne and A.J. Flewitt
}

\begin{abstract}
The utilisation of thin film technology to develop film bulk acoustic resonators (FBARs) and solidly mounted resonators (SMRs), offers great potential to outperform the sensitivity and minimum detection limit of gravimetric sensors. Up to now, the choice between FBARs and SMRs depends primarily on the users' ability to design and fabricate Bragg reflectors and/or membranes, because neither of these two types of resonators has been demonstrated to be superior to the other. In the work reported here, it is shown that identically designed FBARs and SMRs resonating at the same frequency exhibit different responsitivities, $\boldsymbol{R}_{m}$, to mass loadings, being the FBARs more responsive than the SMRs. For the specific device design and resonant frequency $(\sim 2 \mathrm{GHz})$ of the resonators presented, FBARs' mass responsitivity is $\sim 20 \%$ greater than that of SMRs, and although this value should not be taken as universal for all possible device designs, it clearly indicates that FBAR devices should be favoured over SMRs in gravimetric sensing applications.
\end{abstract}

\section{INTRODUCTION}

Bulk acoustic wave (BAW) resonators, such as the quartz crystal microbalance (QCM), are nowadays routinely used in gravimetric sensing applications [1-3]. Historically, the emergence of the quartz crystal as a microbalance can be traced back to Sauerbrey [4], who realised that a resonating quartz crystal could be used for the microgravimetric measurement of sputtered thin films. The addition of a mass on the resonator surface lowers the frequency of resonance, $f_{r}$, due to the increase of the thickness of the resonance cavity and to a variation of the energy confinement due to acoustic impedance mismatch. Hence a variation of the mass bound on the resonators' surface, for example due the adsorption of biological samples, can be detected by tracking changes in $f_{r}$, being the total mass bound on the resonator proportional to the change in $f_{r}$. The mass responsitivity of the sensor, $R_{m}$, is defined as the change in frequency response $\left(\Delta f_{r}\right)$ per unit mass change $(\Delta m)$, as follows:

$$
R_{m}=\frac{\Delta f_{r}}{\Delta m}
$$

If the mass bound on the BAW resonator is small (lower than $\sim 2 \%$ of the total mass of the resonator), there is an approximately quadratic relationship between the fundamental resonant frequency and the mass loading responsitivity [4-6], meaning higher frequency devices will generate exponentially more absolute frequency shift for a given mass load, allowing ever decreasing mass loads to be effectively monitored. QCMs have a mass detection limit of a few nanograms, which is limited by their low operation frequency (5-20 $\mathrm{MHz}$ ) due to the quartz substrate thickness [7,8]. In this respect, the utilisation of thin film technology to develop film bulk acoustic resonators (FBARs) and solidly mounted resonators (SMRs), extremely high frequency QCMs with an operating frequency in the $\mathrm{GHz}$ range, offers great potential to outperform the sensitivity and minimum detection limit of existing QCM-based gravimetric sensors.

The fundamental difference between FBARs and SMRs is the way by which the acoustic energy is trapped within the piezoelectric thin film $[9,10]$. If the piezoelectric film is deposited directly on a substrate, the acoustic wave is mostly transmitted into it due to the similar acoustic impedance between both materials. For the FBAR configuration, the air/piezoelectric interface on both facets of the resonator ensures energy trapping of the acoustic wave regardless of the resonance frequency. In the SMRs, Bragg reflectors consisting of alternating layers of high/low acoustic impedance materials underneath the resonator, effectively trap the acoustic wave. In the last few years, there has been much discussion about which one of these two resonators is the superior one: FBARs are generally regarded as easier to fabricate, and acoustic wave 
trapping occurs at all frequencies thereby allowing maximum design flexibility $[9,11]$. Furthermore the cavity which is etched on the substrate to release the membrane can be utilised to develop a micro fluidic channel system under the resonator, hence enabling integrated lab-on-a-chip biosensors to be developed [1214]. However the membrane is fragile and prone to break in harsh environments. SMRs are significantly more robust than FBARs; however Bragg reflectors must be pre-designed to reflect the desired frequencies, and must be precisely fabricated or else will degrade the effective coupling coefficient as well as creating additional loss mechanisms thus decreasing their quality factor, $Q[15,16]$.

Since there is not an obvious better technology, up to now the choice between FBARs and SMRs depends primarily on the application and the environment that the resonator will be utilised in, but mostly on the users' ability to design and fabricate Bragg reflectors and/or membranes. In the work reported here, it will be shown that FBARs possess greater responsitivity to mass loadings that SMRs and hence this type of BAW resonator is preferred for gravimetric sensing applications.

\section{EXPERIMENTAL}

Both the FBAR and SMR devices presented here consist of a reactively sputtered thin film of $\mathrm{ZnO}(\sim 1.2$ $\mu \mathrm{m})$ sandwiched between metallic electrodes.

The FBARs fabrication process commenced with patterning and sputtering a thin layer $(\sim 100 \mathrm{~nm})$ of $\mathrm{Al}_{2} \mathrm{O}_{3}$ on the bottom of a double-side polished (100)oriented Si substrate. This layer acts as a hard mask on a forthcoming deep reactive ion etching (DRIE) process and defines the dimensions of the membrane. A second $\mathrm{Al}_{2} \mathrm{O}_{3}$ layer $(100 \mathrm{~nm})$, that doubles as a support for the $\mathrm{ZnO}$ membrane and an etch barrier for the forthcoming DRIE process, was reactively sputtered on the top of the substrate. The SMRs fabrication process commenced with growing seven alternating low and high acoustic impedance layers of porous $\mathrm{SiO}_{2}(669 \mathrm{~nm})$ and $\mathrm{Mo}(786$ $\mathrm{nm})$ on a $\mathrm{Si}(100)$ substrate, to form an acoustic mirror centred at $\sim 2 \mathrm{GHz}$ and with $\sim 1 \mathrm{GHz}$ bandwidth. For both the FBARs and SMRs, the bottom electrodes $(4 / 50$ $\mathrm{nm} \mathrm{Cr} / \mathrm{Au}$ ) were patterned through a standard photolithography process and thermally evaporated on the top $\mathrm{Al}_{2} \mathrm{O}_{3}$ layer (for the FBARs) or the top $\mathrm{SiO}_{2}$ layer (for the SMRs). The $\mathrm{ZnO}$ piezoelectric films were then reactively sputtered from a 4 -in diameter metallic $\mathrm{Zn}$ target in a high target utilization sputtering (HiTUS) system [17]. The $\mathrm{ZnO}$ films were sputtered with a 10:7 Ar: $\mathrm{O}_{2}$ admixture at a total pressure of $\sim 3 \times 10^{-3} \mathrm{mbar}$, and at room temperature. These conditions provided deposition rates of $\sim 50 \mathrm{~nm} \cdot \mathrm{min}^{-1}$. Further information on the sputtering conditions and characterisation of the resulting $\mathrm{ZnO}$ films is reported elsewhere [18-20].

$\mathrm{X}$-ray diffractometry (XRD) using $\mathrm{CuK}_{\alpha}$ radiation was used to assess the crystal quality of the sputtered $\mathrm{ZnO}$ films. The $\theta-2 \theta$ scan of the films over a broad angle range $\left(2 \theta=30^{\circ}-80^{\circ}\right)$ confirmed the preferential orientation of the films along the (0001) direction ( $c$ axis). $\theta-2 \theta$ scans are shown in Fig. 1(a) where only the peaks corresponding to the (0002) and (0004) $\mathrm{ZnO}$ planes, together with those of the (004) Si planes and (110) Mo planes (for the films grown on the reflector only) are observed. The identification of the peaks is based on their tabulated values [21].

Fig. 1(b) presents the rocking curves of the most intense reflection from $\mathrm{ZnO}$, i.e., the (0002) peak at $\theta=$ $17.1^{\circ}$. The peaks have a full width at half maximum (FWHM) of $5.7^{\circ}$ and $9.1^{\circ}$ for the films deposited on $\mathrm{Al}_{2} \mathrm{O}_{3}$ and Bragg reflector respectively, indicating the smaller angular dispersion of the crystallites around the $c$-axis of the films utilised to fabricate the FBARs. Nevertheless both FWHM values ensure sufficient piezoelectricity for good resonators' performance.

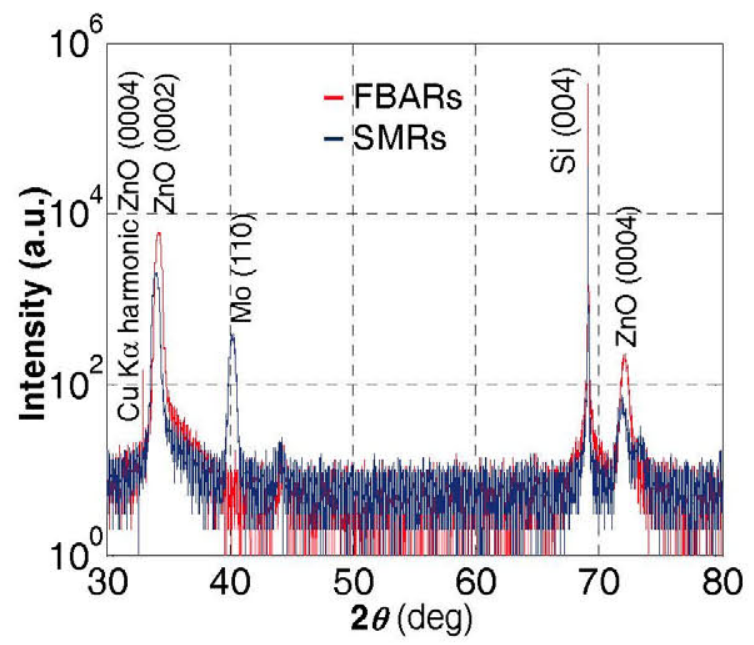

(a)

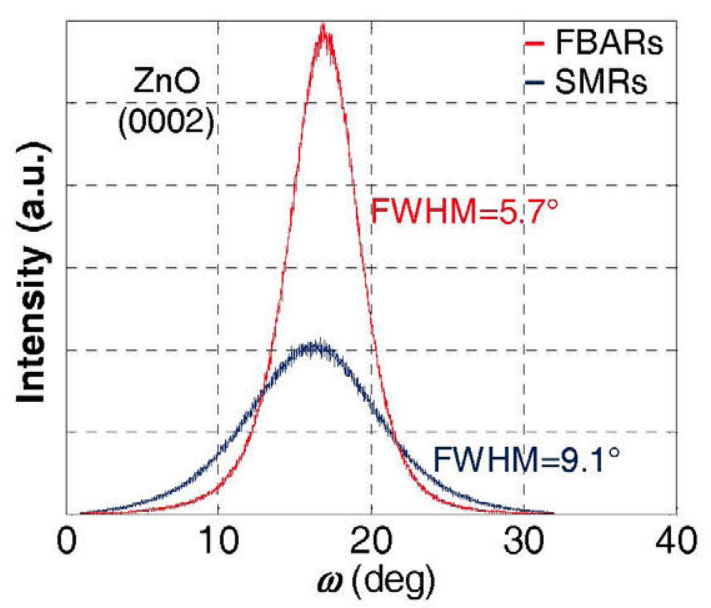

(b)

FIG. 1. (a) X-ray diffractograms in a $\theta-2 \theta$ configuration of the $\mathrm{ZnO}$ films deposited on $\mathrm{Al}_{2} \mathrm{O}_{3}$ for $\mathrm{FBAR}$ fabrication (red line) and on Bragg reflector for SMR fabrication (blue line). (b) Rocking curves of the $\mathrm{ZnO}(0002)$ peaks.

After $\mathrm{ZnO}$ deposition, the top electrode was patterned with a lift-off photolithography process with 
identical materials and thicknesses to the bottom electrode. Via etch holes were then formed through the $\mathrm{ZnO}$ for electrical connection to the bottom electrode by wet etching the $\mathrm{ZnO}$ in a $2 \%$ glacial acetic and phosphoric acids solution at room temperature. Finally the $\mathrm{Si}$ from the back of the wafer was removed on the FBAR devices with a DRIE process to release the $\mathrm{ZnO} / \mathrm{Al}_{2} \mathrm{O}_{3}$ membrane, and the $\mathrm{Al}_{2} \mathrm{O}_{3}$ layer under the $\mathrm{ZnO}$ was removed by wet etching in potassium hydroxide at room temperature. Fig. 2 shows a top view of the devices fabricated. The resonant area is where the top and bottom electrodes overlap.

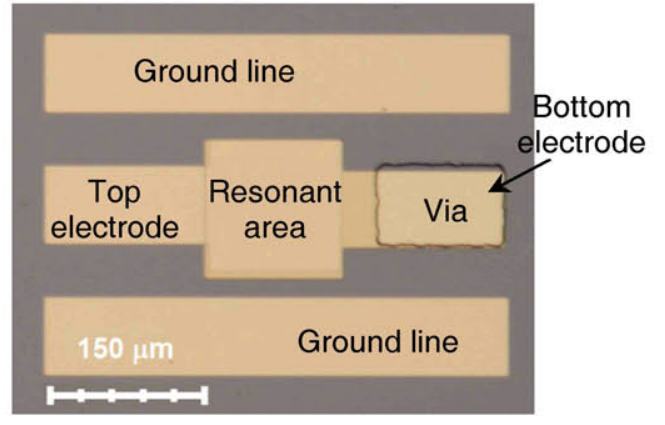

FIG. 2. Top view of the devices fabricated.

\section{RESULTS AND DISCUSSION}

\section{A. Resonators' design and electrical characterisation}

The transmission characteristics of the devices were measured with a coplanar probe station (on a GSG configuration) connected to a network analyser. Both types of devices were found to resonate at $\sim 1.94 \mathrm{GHz}$, which lies well within the optimum range of the reflector's performance.

The quality factors $(Q)$ at the parallel resonant frequency were extracted from the S-parameter spectrum using the IEEE standard definition [22]:

$$
Q=\frac{1}{2} f_{a-r} \frac{\partial \varphi}{\partial f}
$$

where $\varphi$ represents the admittance phase and $f_{a-r}$ represents the anti-resonance frequency. $Q$ was found to be $\sim 1380$ and $\sim 840$ for the FBARs and SMRs respectively. The lower value of $Q$ exhibited by the SMRs is explained in part by the slightly worse $\mathrm{ZnO}$ crystallographic orientation (as indicated by the wider XRD rocking curve).

\section{B. Comparison of the mass responsitivities of FBARs and SMRS}

When an additional mass is added onto a resonator's surface, a negative frequency shift will be observed on its frequency response. This has been verified experimentally by sputtering thin aluminium layers of different thicknesses on top of the resonators. In order to calculate precisely the frequency shifts due to different mass loadings, an automated measurement routine was written with LabVIEW® to continuously monitor and record the resonance spectrum of the resonators. Firstly the resonant frequency of unloaded devices was verified to be stable (to $\pm 2 \mathrm{kHz}$ ) by tracking their response over a period of $\sim 15 \mathrm{~min}$. Then layers of $\mathrm{Al}$ of different thicknesses were evaporated on top of identical devices and the exact mass load being added was calculated from the additional layer thickness, lateral dimensions of the resonant area and the aluminium's mass density. The resonators were then re-measured and their resonant frequency was again tracked over several minutes to verify stability. $\Delta f_{r}$ was calculated by comparison of $f_{r}$ s of loaded and unloaded resonators.

The environmental temperature, which is known to affect the frequency response of the devices [23-25], was controlled by placing the resonators, wire bonded to $50 \Omega$ transmission line PCBs, into a solid brass environmental isolation assembly with a high thermal mass. This assembly was placed onto the metallic output pads of a HAAKE K20 water bath, which pumped a thermal fluid at fixed rate and controllable temperature. This was able to control the temperature of the resonators within less than $\pm 0.5^{\circ} \mathrm{C}$ of the set temperature as measured by an automated thermocouple (National Instruments TC 01 ) with a resolution of $0.1^{\circ} \mathrm{C}$ and a response time of less than 1 second. All measurements were carried out at a constant temperature of $22^{\circ} \mathrm{C}$.

The $f_{r}$ of the two types of resonators decreases linearly due to mass loads within the range studied. The $\Delta f_{r}$ observed for different loads is shown in Fig. 3 together with finite element analysis (FEA) predictions, where it was assumed that the additional mass added was evenly distributed on the resonators' surface.

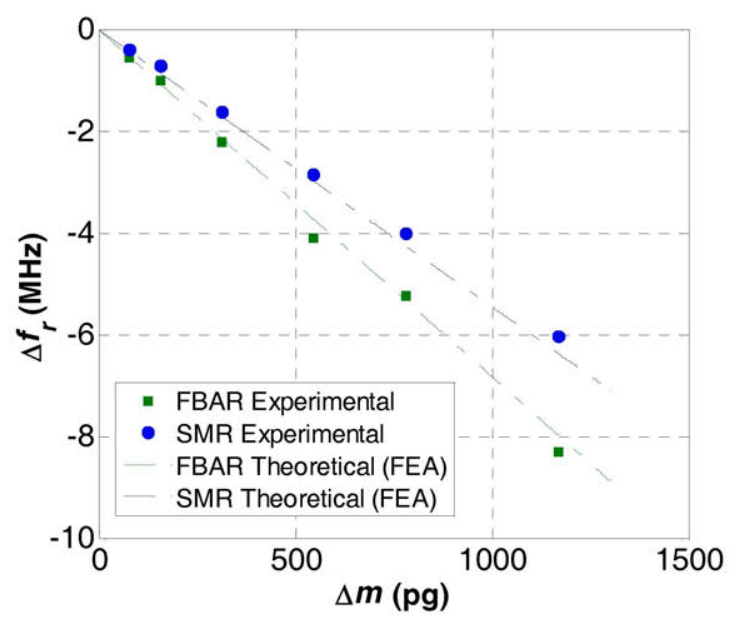

FIG. 3. Experimentally observed variation of the frequency of resonance, $\Delta f_{r}$, at room temperature $\left(22^{\circ} \mathrm{C}\right)$ when different mass loads are deposited on the FBARs and SMRs surface. The dashed lines represent theoretical (FEA) calculations.

It can be seen that the FBARs exhibit a greater responsitivity, $R_{m}=\sim 6.9 \mathrm{kHz} \cdot \mathrm{pg}^{-1}$, than the SMRs, $R_{m}=\sim 5.1 \mathrm{kHz} \cdot \mathrm{pg}^{-1}$. It is worthwhile noting that the precise values of responsitivity depend in part on the 
resonators' size and design, and therefore these specific values can not be taken as an universal FBAR nor SMR response to mass loadings. However this result is very significant in that identically designed FBARs and SMRs resonating at the same frequency exhibit different frequency shifts for identical mass loads being the FBARs more responsive than the SMRs.

\section{CONCLUSIONS}

Evaluation of the mass responsitivity of FBARs and SMRs has been carried out. Identical sets of FBARs and SMRs were fabricated with their fundamental resonant mode at $\sim 2 \mathrm{GHz}$. In order to calculate the responsitivity of both types of devices, thin aluminium layers of different thicknesses were evaporated on top of the FBARs and SMRs. The exact mass load being added, $\Delta m$, was precisely calculated from the additional layer thickness, lateral dimensions of the resonant area and the aluminium's mass density. The $f_{r}$ of the two types of resonators decreases linearly due to mass loads within the range studied, but it was found that FBARs exhibit a greater responsitivity, $R_{m}=\sim 6.9 \mathrm{kHz} \cdot \mathrm{pg}^{-1}$, than the SMRs, $R_{m}=\sim 5.1 \mathrm{kHz} \cdot \mathrm{pg}^{-1}$. However these specific values of responsitivity depend on the resonators' design and frequency of resonance, and therefore should not be taken as universal FBAR nor SMR responses to mass loadings.

\section{ACKNOWLEDGEMENTS}

The work reported here was supported in part by the EPSRC, grants number EP/F063865/1 and EP/F06294X/1. L. García-Gancedo acknowledges support from the National Natural Science Foundation of China (NSFC) through grant number 61150110485 . J. Pedrós acknowledges the support from the Moncloa Campus of International Excellence (UPM-UCM, ISOM). E. Iborra, M. Clement, J. Olivares and J. Capilla acknowledge financial support from the Ministerio de Ciencia e Innovación of Spain through project MAT2010-18933, and from the European Union through the European Regional Development Fund (FEDER).

\section{REFERENCES}

[1] G. García-Martinez, E. Alonso-Bustabad et al., 'Development of a mass sensitive quartz crystal microbalance (QCM)-based DNA biosensor using a $50 \mathrm{MHz}$ electronic oscillator circuit', Sensors, Vol. 11, pp. 7656-7664 (2011)

[2] L. García-Gancedo, Z. Zhu et al., 'AlN-based BAW resonators with CNT electrodes for gravimetric biosensing', Sens. Act. B: Chem., Vol. 160, pp. 1386- 1393 (2011)

[3] X.B. Zhao, G.M. Ashley et al., 'Protein functionalized $\mathrm{ZnO}$ thin film bulk acoustic resonator as an odorant biosensor', Sens. Act. B: Chem., Vol. 163, pp. 242-246 (2012)

[4] G. Sauerbrey, 'The use of quartz oscillators for weighing thin layers and microweighing' (in German), Z. Physik, Vol. 155, pp. 206-222 (1959)

[5] R. Gabl, H.-D. Feucht et al., 'First results on label-free detection of DNA and protein molecules using a novel integrated sensor technology based on gravimetric detection principles', Biosens. Bioelectron., Vol. 19, pp. 615-620 (2004)

[6] D. S. Ballantine, R.M. White et al., 'Acoustic Wave Sensors: Theory, Design, and Physico-Chemical Applications' Academic Press (1996)

[7] A. Wajid, 'On the accuracy of quartz crystal microbalance (QCM) in thin film depositions', Sens. Act. A: Phys., Vol. 63, pp. 41-46 (1997)

[8] C.I. Cheng, Y.-P. Chang et al., 'Biomolecular interactions and tools for their recognition: focus on the quartz crystal microbalance and its diverse surface chemistries and applications', Chem. Soc. Rev., Vol. 41, pp. 1947-1971 (2012)

[9] R. Ruby, 'Review and comparison of bulk acoustic wave FBAR, SMR technology', IEEE Ultrason. Symp., pp. 1029$1040(2007)$

[10] H. Campanella, 'Acoustic wave and electromechanical resonators', Artech House (2010)

[11] J.H. Jung, Y.H. Lee et al., 'Vibration Mode Analysis of RF Film Bulk Acoustic Wave Resonator using Finite Element Method', IEEE Ultrason. Symp., pp. 847-850 (2001)

[12] G. Wingqvist, 'AlN-based sputter-deposited shear mode thin film bulk acoustic resonator (FBAR) for biosensor applications - A review', Surf. Coat. Tech., Vol. 205, 1279-1286 (2010)

[13] I. Katardjiev and V. Yantchev, 'Recent developments in thin film electro-acoustic technology for biosensor applications', Vacuum, Vol. 86, pp. 520-531 (2012)

[14] G. Wingqvist, J. Bjurstrom et al., 'Shear mode AlN thin film electro-acoustic resonant sensor operation in viscous media', Sens. Act. B: Chem., Vol. 123, pp. 466-473 (2007)

[15] J.M. 1e Floch, M.E. Tobar et al., 'Low-loss materials for high Q-factor Bragg reflector resonators', Appl. Phys. Lett., Vol. 92, 032901 (2008)

[16] T. Pensala, R. Thalhammer et al., 'Experimental investigation of acoustic substrate losses in $1850-M H z$ thin film $B A W$ resonators', IEEE Trans. Ultras. Ferro. Freq. Contr., Vol. 56, pp. 2544-2552 (2009)

[17] A.J. Flewitt, J.D. Dutson et al., 'Stability of thin film transistors incorporating a zinc oxide or indium zinc oxide channel deposited by a high rate sputtering process', Semicond. Sci. Technol., Vol. 24, 085002 (2009)

[18] L. García-Gancedo, J. Pedrós et al., 'Ultrafast sputtered ZnO thin films with high $k_{T}$ for acoustic wave device applications', IEEE Ultrason. Symp., pp. 1064-1067 (2010)

[19] J. Pedrós, L. García-Gancedo et al., 'Guided propagation of surface acoustic waves and piezoelectric field enhancement in ZnO/GaAs systems', J. Appl. Phys., Vol. 110, 103501 (2011)

[20] L. García-Gancedo, J. Pedrós et al., 'Room-temperature remote-plasma sputtering of c-axis oriented zinc oxide thin films', J. Appl. Phys., Vol. 112, 014907 (2012)

[21] International Centre for Diffraction Data (ICDD), files Nos 361451, 27-1402 and 04-0809 for $\mathrm{ZnO}, \mathrm{Si}$ and Mo respectively.

[22] IEEE standard on piezoelectricity, ANSI/IEEE Std. 176 (1987)

[23] S. Rai, Y. Su et al., 'A digitally compensated $1.5 \mathrm{GHz}$ CMOS/FBAR frequency reference', IEEE Trans. U1tras. Ferro. Freq. Contr., Vol. 57, pp. 552-561 (2010)

[24] J. Bjurstrom, G. Wingqvist et al., 'Temperature compensation of liquid FBAR sensors', J. Micromech. Microeng., Vol. 17, pp. 651-658 (2007)

[25] L. García-Gancedo, J. Pedrós et al., 'Dual mode thin film bulk acoustic wave resonators for parallel sensing of temperature and mass loading', Bios. Bioelectron., Vol. 38, pp. 369-374 (2012) 